\title{
Prediction of Regulatory Networks of PITX2 Gene Expression in Mandibular Asymmetry Related to Oral Muscle Function
}

\author{
Ervina Sofyanti \\ Doctoral Program \\ Faculty of Dentistry, Universitas Sumatera Utara \\ Medan, Indonesia \\ ervina.sofyanti@usu.ac.id \\ Tiar Pratamawati \\ Department of Genetics \\ Medical Faculty, Universitas Swadaya Gunung Djati \\ Cirebon, Indonesia \\ dr.tyar@yahoo.co.id
}

\author{
Trelia Boel \\ Department of Dentomaxillofacial Radiograph \\ Faculty of Dentistry, Universitas Sumatera Utara \\ Medan, Indonesia \\ trelia.boel@usu.ac.id \\ Elza Ibrahim Auerkari \\ Department of Oral Biology \\ Faculty of Dentistry, University Indonesia \\ Jakarta, Indonesia \\ eauerkari@yahoo.com
}

\begin{abstract}
The teeth, muscles (associated nerves) and joints are considered as an interdependent unit in neuromuscular dentistry component. The embryonic development of the temporomandibular joint (TMJ) differs considerably from other synovial joints. Condylar cartilage is an important site of mandibular growth and it forms part of the TMJ. The issue of occlusal characteristics asymmetry related to (TMJ) function and poor posture still remains debatable in neuromuscular dentistry. Recent studies reported that gene expression of specific growth factors and other signaling molecules have been involved in condylar growth. PITX2 is an active gene in the nodal pathway that determines left-right asymmetry in vertebrae during embryogenesis and expressed in satellite cells of adult human skeletal muscle. There were several mechanisms by which PITX2 isoforms may interact to both activate and repress gene expression in brain, craniofacial region, and pituitary. The effects of asymmetric nodal pathway gene expression are well documented in embryonic development for first branchial arch structures differences as potential candidate genes for facial asymmetry. Overall, a molecular basis for differential gene regulation through the expression of PITX2 gene isoform can provide genetic differences in oral muscle function when mandibular asymmetry is present or absent in skeletal malocclusion. This paper aims to help the clinician in understanding the biomolecular site that contribute in unique features of mandibular asymmetry related to oral muscle function.
\end{abstract}

Keywords-PITX2 gene, mandibular asymmetry, oral muscle, neuromuscular

\section{INTRODUCTION}

Neurons communicate to muscle cells and maintain proper clustering of the proteins required for transmission in the postsynaptic muscle. In neuromuscular dentistry, the teeth, muscles (associated nerves) and joints are considered as an interdependent unit. Skeletal muscle's primary function is to generate force and produce movement. This requires coordination among many physiological pathways and their associated components. The effect of mechanical, biological, and chemical stimuli altered transcriptional pathways in loss of skeletal muscle function [1].

The skeletal phenotype can be detected by radiograph analysis in $2 \mathrm{D}$ and $3 \mathrm{D}$ measurements. The phenotype and genotype analysis in condyle region should be provided as diagnostic biomarker for ancillary method in orthodontic diagnosis nowadays. The relative importance of environment versus hereditary has been controversial since the days of Angle. Some studies reported rapid advance of heritability of malocclusion; however the study regarding the genetic contributions to asymmetry is rare $[2,3]$. The size of corpus, ramus, and mandibular condyle, as well as the timing and amount of condylar growth vary considerably between individuals. The development of molecular basis for differential gene regulation through some gene expression can provide genetic differences when mandibular asymmetry is present or absent in any skeletal malocclusions. The proper diagnosis is required for addressing treatment limitations and therapeutic options because some misdiagnosis in some asymmetry cases can result in erroneous treatment plans that will end in frustration for both patient and orthodontist [4]. A better understanding of the genetic variables, especially contribute to asymmetry mandible is necessary to develop new preventive strategies and allow the clinician to select the early courses of any dental treatment to prevent the development of any occlusal interferences. So, the aim of this paper to help the clinician in understanding the biomolecular site that contribute in unique features of mandibular asymmetry related to oral muscle function. 


\section{LITERATURE REVIEW}

\section{A. Mandibular asymmetry}

Mandibular asymmetry is a common craniofacial deformity that resulted from asymmetric growth of the mandible or other certain disease affecting the facial growth. It has a direct effect on the quality of life due to functional problems of the stomatognathic system (TMDs) and esthetic concern in any type of malocclusion [5,6]. The National Dental Centre of Singapore reported that asymmetry occurs in $8 \%$ of skeletal class II and in 50\% of skeletal class III individuals among a multiracial population [7]. In contrast, the prevalence of asymmetry in skeletal class II is $28 \%$ and $40 \%$ for skeletal class III in a dentofacial deformities population undergoing surgical corrections at the University of North Carolina [8]. The proportion of mandibular asymmetry was reported $32.9 \%$ in $8-30$ year old subjects among the multiracial Jakarta population in Indonesia whilst $25.2 \%$ in soft tissue mandibular asymmetry and $14.1 \%$ in skeletal [8]. The variance data because asymmetry occurs through genetic and functional differences during growth and development, heritable background influence the proportion of sagittal, vertical and transverse dysplasia present in a local population should be studied [3]. Then, factors potentially contributing to this individual variation are also the extent of masticatory action related to the consistency of the diet and genetic predisposition.

The development of various skeletal mandibular is a multifactorial interaction during morphogenetic period of the mandible that might be possible has been regulated during infancy. The embryonic development of the temporomandibular joint differs considerably from other synovial joints. Condylar cartilage is an important site of mandibular growth and it forms part of the temporomandibular joint as well. The condylar cartilage is categorized as secondary cartilage that has distinct biological characteristics. Mechanical loading of functional stimuli might influence the responses of the condylar cartilage and the subsequent growth of the mandible. Some studies have documented the influences of various genes that are involved in the regulation of mandibular morphogenesis, such as: gene expression of specific growth factors or other signaling molecules that are involved in condylar growth $[9,10]$.

\section{B. Neuromuscular dentristry}

The etiology of muscle activity that influenced bone size and density has not yet fully been understood. Bite force is associated with muscle cross sectional area and mechanical advantage, but muscle size is not always associated with facial skeletal form. This work has demonstrated that variations in area and size of fiber types contribute to differences in the vertical dimension of facial growth. Bilateral comparisons of masseter muscle fiber types made in subjects with or without facial asymmetry show no differences with symmetry, but significant differences are found between sides when asymmetry is present [9].
The muscle movement can produce the joint cavitation to separate the connective tissue by rupturing coalesce spaces to coalesce into functional cavities. The skeletal distortion might be happen in early immobilization of developing joints results in absence of joints cavities and fusion of the articulations. Failure of cavitation of one or both joint compartments results in ankyloses that may be unilateral or bilateral [10]. The unilateral activities lead to asymmetry mandible that results in imbalanced growth and function of TMJ and associated muscles. The mandibular asymmetry is becoming an issue in any dental treatment since the morphological diversity and variations of condylar shape are related to muscle coordination, especially for orthodontist [11].

The issue of occlusal characteristics asymmetry related to temporomandibular joint (TMJ) function and poor posture still remains debatable in neuromuscular dentistry. The teeth malocclusions, wrong posture, and ocular divergences disorders were also reported had and appear to request a multidisciplinary medical approach in treatment of $13 \%(\mathrm{n}=70)$ primary school students in Genoa. The existence of causal connections can stimulate skeletal and muscle development of any organ in vertebrae. Then this might be considered as a risk factor in developmental asymmetry [12]. Any dysfunction, occlusal disorder, postural abnormality or trauma upper quarter could likely lead to a problem at adjacent or related components. For this reason, an evaluation of cervical spine must be carried out on TMD patients. Various studies have suggested that there is a positive correlation between TMD and poor posture with mandibular asymmetry as one of occlusal disease sign [13].

\section{Pituitary Homeobox-Type 2 (PITX2)}

PITX2 is a gene active in the Nodal Pathway that determines left-right asymmetry in vertebrae during embryogenesis [14] and has recently been reported to be expressed in satellite cells of adult human skeletal muscle [3]. The term of satellite cells is all skeletal muscle which is able to regenerate following injury due to the presence of myogenic stem cells. PITX2 gene is a paired-related homeobox gene and was identified by positional cloning of the $4 \mathrm{q} 25$ locus in reverse strand of $110,617,423$ bp to $110,642,123 b p$ [16].

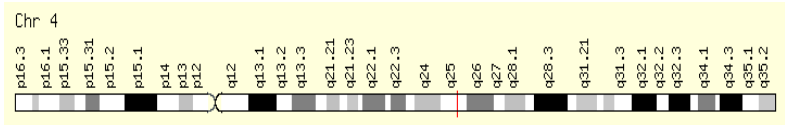

Figure 1. PITX2 gene.

PITX2 or Paired-like homeodomain transcription factor 2 is a bicoid-like homeobox transcription factor that plays an important role in morphogenesis. Distinct transcription factors are required for the early determination, development, and maintenance of extraocular muscles (EOM) compared to limb skeletal muscle. It was also required for the maintenance of characteristic properties of the adult EOM phenotype 
[17,18]. PITX2 gene expression differences was reported to contributed in Nodal Signaling Pathway (NSP) which pattern the left-right axis for both skeletal and muscle development of facial asymmetry, especially in posterior region [3].

Laterality (left-right-sidedness) also is established in early embryonic stage, development and orchestrated by a cascade of signal molecules and PITX2 genes (Figure 1). When the primitive streak appears, FGF8 is secreted by cells in the node and primitive streak and induces expression of Nodal, but only on the left side of the embryo. Later, as the neural plate is established, FGF8 maintains nodal expression in the lateral plate mesoderm, as well as lefty-2, and both of these genes upregulate PITX2. PITX2 is a homeobox-containing transcription factor responsible for establishing leftsidedness and its expression is repeated on the left side of the heart, stomach, and gut primordial as these organs are assuming their normal asymmetrical body positions. If the gene is expressed ectopically (e.g., on the right side), this abnormal expression results in laterality defects, including situs inversus, dextrocardia (placement of the heart to the right side) and a variety of heart defects. Simultaneously, lefty is expressed on the left side of the floor plate of the neural tube and may act as a barrier to prevent left-sided signals from crossing over [15].

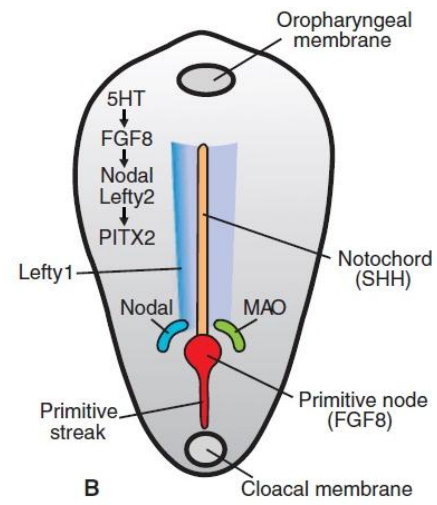

Figure 2. Gene expression patterns responsible for establishing the left-right body axis

The PITX2 genes are members of the bicoid class of the homeodomain genes as key regulators in development process and to be expressed in the brain, heart, pituitary, mandibular and maxillary region, eye, and umbilicus. In human, PITX2 gene mutations has been reported as a contributing cause of haplo insufficiency condition affecting the eyes, teeth and umbilicus with associated abnormalities of the craniofacial region (midfacial hypoplasia, prognathism) $[18,19]$ and are present in individuals with AxenfeldRieger Syndrome, Peters' anomaly, iridogoniodysgenesis, iris hypoplasia, and CHARGE syndrome [20-22]. The genetic hierarchies of PITX2gene in control muscle development during embryogenesis are found in trunk-limb and headmuscle development. This gene also emerged as a key transcription factor that modulated adult myogenesis
[18]. Kamnasaran et al (2003) reported a child with the translocation $\mathrm{t}(4 ; 14)(\mathrm{q} 25 ; \mathrm{q} 13)$ had two molecular breaks on $4 \mathrm{q} 25$ and had mild craniofacial dimorphism and agenesis of the corpus callosum without limb or ocular abnormalities [23]. The etiology of mandibular asymmetry is vast and a combination of genetic and environmental factors. The myogenic problems are one of the common causes in mandibular asymmetry related to Nodal signaling pathway (NSP) genes in masseter muscles [3].

There are PITX2 isoforms that are produced by alternative splicing and use of different promoters. PITX2A and PITX2B are generated by alternative splicing mechanisms, and PITX2C uses an alternative promoter located upstream of exon 4. The PITX2D isoforms acts to down-regulate the transcriptional activities of PITX2A and PITX2C. The expression of PITX2 isoforms can greatly influence gene expression [14]. PITX2D was identified as the new PITX2 isoform (PITX2D) that generated by the PITX2C alternative promoter and differential splicing was found in human. This isoform was expressed in humans and identified from a human craniofacial library. The PITX2D isoform acts to down-regulate the transcriptional activities of PITX2A and PITX2C. All isoforms can form homodimers, and heterodimers are formed with PITX2B. In the brain, craniofacial region, and pituitary, which express all three major PITX2 isoforms, the interactions between PITX2 isoforms provides a mechanism to tightly regulate gene expression controlled by PITX2 and provided several mechanisms by which PITX2 isoforms may interact to both activate and repress gene expression. The molecular basis for organ/tissue development by PITX2 isoforms has been provided by the greatly influenced of PITX2 isoforms gene expression. Furthermore, the evidence for the regulation of PITX2 isoform transcriptional activity in a cell-dependent manner with mechanism for the regulation of PITX2 transcriptional activation through the action of a novel PITX2 isoform [14]. These reports establish that PITX2 family of bicoid-like homeodomain genes are key regulators of important development processes and are required to regulate specific genes. PITX2 isoforms provided a molecular basis for organ/tissue development (Figure 3).

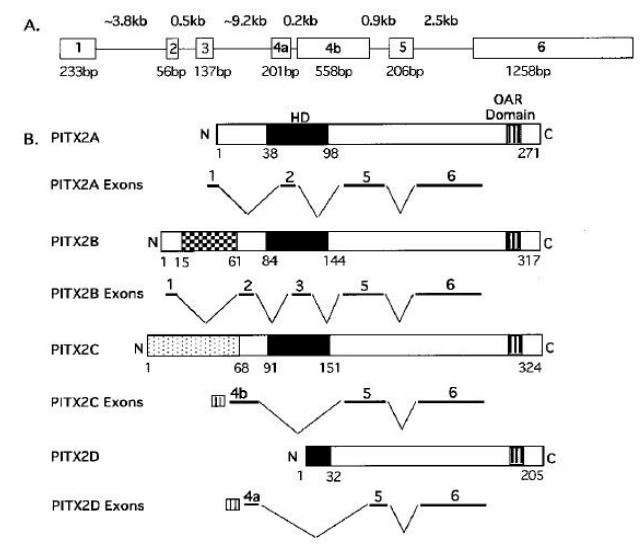

Figure 3. The initial PITX2 major isoforms found in humans. 
Based on the ocular, cardiac, and craniofacial expression pattern of PITX2 gene and the pleiotropic effects of loss PITX2 function in both mouse and human, Martin et al., (2002) hypothesized the PITX2 mutations may contribute to the multiple phenotypic anomalies present in individual with CHARGE. By direct sequencing of DNA from 29 affected individuals, there were no mutations in PITX2, except sequence polymorphisms (missense mutation) in CHARGE syndrome for two subjects [19]. The second promoter was found in exon 4 of PITX2C gene. Unlike the other PITX2 isoforms, PITX2D did not bind DNA and showed no transcriptional activity. However, when coexpressed, PITX2D suppressed the transcriptional activities of the other PITX2 isoforms [14].

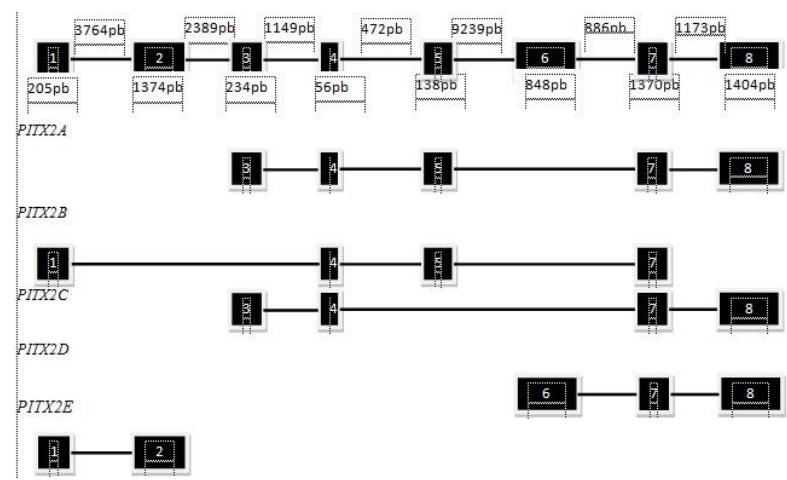

Figure 4. Five polymerase chain reaction products (A-E) and sizes in base pairs of PITX2 gene.

PITX2 is especially important to development of mesoderm-derived first branchial arch structures. In addition to lateral plate mesoderm, PITX2 is also expressed in cephalic and first branchial arch mesoderm during gastrulation. In the first arch PITX2 is necessary to establish pre-myoblast specification of mesoderm and induction of ectoderm for tooth bud formation. Masticatory muscles are absent in PITX2 mutant mice, while muscles in the other branchial arches develop, but have some significant deformations. Lack of skeletal structure development also occurs, but is limited to the first arch. This suggests that normal PITX2 expression is essential for induction of jaw development. Abnormal PITX2 signaling is well established before migration of neural crest cells into the arch, which disrupts normal organogenesis of the jaws. After organogenesis is completed PITX2 continues to be expressed in most muscles of the head and trunk, and persists into adulthood. Similar experiments demonstrate that PITX2 expression is required for normal functioning of adult extraocular muscles $[3,18]$.

\section{DISCUSSION}

Understanding the biology of underlying craniofacial growth and dental relations related to asymmetry as a fundamental for getting proper diagnosis. The genetic component was reported higher for skeletal pattern than for dental features in any malocclusion. The genetic proof was believed directly related to the diagnosis of familial dentofacial problems, included mandibular asymmetry. Modern orthodontists need to be aware of the basis of the genetic sciences, recent advances in the genetic researches and application in orthodontic practices. The complexity of genetics in dentofacial variation explains in part why most treatment approaching malocclusion with mandibular asymmetry is directed to the symptoms rather than its etiology. Despite this complexity, the study of etiology is fundamental for any clinicians in. It will also provide future potential for making significant impacts in clinical practice in order to obtain stability of any dental treatment result. Some studies reported that genetic component of variability for measurements in vertical dimension were stronger than sagittal dimension [2,24]. The mechanism of bite force from the mandible to cranium while functioning suggest the magnitude of joint loading overtime related to condylar size, although it can be affected by the other risks factors, such as: type of skeletal and dental malocclusion, temporomandibular disorder and bad habit.

Previously, PITX2 locus produces three isoform designated PITX2A-2C that arises through differential splicing and alternative promoter usage. Then the specific promoter in exon of PITX2C gene resulted isoform of PITX2D and PITX2E gene. The different isoforms are generated by both alternative splicing and alternative promoter usage and have both overlapping and distinct expression patterns [14. These all PITX2 isoforms are expressed in proliferating satellite cell derived myoblasts in adult myogenesis as key transcription modulator [22]. Identification of gene expression in Nodal Signaling Pathway of PITX2 expression gene was also reported to help promote development of asymmetry because this gene plays a central role in left right asymmetry [3]. The studies about correlation of Temporomandibular Disorder sign and symptom (TMDs) to mandibular asymmetry is a debatable issue nowadays because the etiologic factors for both conditions are obviously multifactorial and related to oral muscle function $[13,25]$.

As a conclusion, a molecular basis for differential gene regulation through the expression of PITX2 gene isoform can provide genetic differences when mandibular asymmetry is present or absent in skeletal malocclusions. The effects of asymmetric nodal pathway gene expression that are well documented in embryonic developmental for first branchial arch structures differences was potential candidate genes for facial asymmetry in adult. Further studies will be required for understanding the unique features of mandibular asymmetry related to oral muscle function.

\section{REFERENCES}

[1] L.R Smith, G. Mayor, R.L Lieber, "Systems analysis of biological networks in skeletal muscle function," Wiley Interdiscip. Rev. Syst. Biol. Med., vol. 5, pp. 55-71, Nov 2012.

[2] D.G. Cakan, F. Ulkur, T.U. Taner, "The genetic basis of facia skeletal characteristics and its relation with orthodontics," Eur. J. Dent., vol. 6, pp. 340-345, July 2012

[3] R. Nicot, M. Hottenstein, G. Raoul, J. Ferri, M. Horton, J.W. Tobias, et al., "Nodal pathway genes are down-regulated in 
facial asymmetry,” J. Craniofac. Surg., vol. 25, pp. 548-555, November 2014.

[4] D.C. Van Elslande, S.J. Russett, P.W. Major, C. Flores-Mir, "Mandibular asymmetry diagnosis with panoramic imaging," Am. J. Orthod. Dentofacial Orthop., vol. 132, pp. 183-192, August 2008.

[5] O.S. Sezgin, P. Celenk, S. Arici, "Mandibular asymmetry in different occlusion patterns," Angle Orthod., vol. 77 pp. 803807, September 2007.

[6] H. Lin, P. Zhu, Y. Lin, S. Wan, X. Shu, Y. Xu, et al., "Mandibular asymmetry: a three-dimensional quantification of bilateral condyles," Head Face Med., vol. 9, pp. 42, December 2013.

[7] M.T. Chew, "Spectrum and management of dentofacial deformities in a multiethnic Asian population," Angle Orthod., vol.76, pp. 806-809, September 2006

[8] M. Purbiati, M.K. Purwanegara, L. Kusdhany, "Prediction of Mandibulofacial Asymmetry using Risk Factor Index and Model of Dentocraniofacial Morphological Pattern," J. Int. Dent. Med. Res., vol. 9, pp. 195-201, 2016.

[9] F. Xue, R.W. Wong A.B. Rabie, "Genes, genetics, and Class III malocclusion," Orthod. Craniofac. Res., vol. 13, pp. 69-74, May 2010.

[10] S.M. Sperber, G.D. Guttman, Mechanism of embryology. In: Craniofacial embryogenetics and development, $2^{\text {nd }}$ ed., vol. 2. People's Medical Publishing House: Connecticut, 2010, pp. 149-165.

[11] K Durgha, "Condylar Morphology- A Review," IOSR-JDMS., vol. 13, pp. 57-59, July 2014

[12] F. Silvestrini-Biavati, A. Ugolini, N. Laffi, C. Canevello, A. Silvestrini-Biavati, "Early diagnostic evaluation of mandibular symmetry using orthopantomogram," Indian J. Dent. Res., vol. 25, pp. 154-159, March 2014

[13] Khan,"Neuromuscular dentistry: Occlusal diseases and posture," Int. J. Dent. Oral Sci., vol. 3, pp. 348-352, October 2016.

[14] C.J. Cox, H.M. Espinoza, B. McWilliams, K. Chappell, L. Morton, T.A. Hjalt, et al., "Differential regulation of gene expression by PITX2 isoforms," J. Biol. Chem., vol. 12, pp. 25001-25010, July 2002.

[15] T.W. Sadler, Langman's medical embriology, 12th ed., China: Lippincott Williams\&Wilkins, 2012, pp. 51-58.

[16] (2017, August 28) Gene: PITX2 ENSG00000164093. Available: http://aug2017.archive.ensembl.org/Homo_sapiens/Gene/Summ ary? $/ \mathrm{db}=$ core; $\mathrm{g}=\mathrm{ENSG} 00000164093 ; \mathrm{r}=4: 110617423$ 110642123 .

[17] S.L. Hebert, M. L. Daniel, L.K McLoon, "The role of PITX2 in maintaining the phenotype of myogenic precursor cells in the extraocular muscles," PLoS One, vol. 8, pp. e58405, March 2013.

[18] F. Hernandez-Torres, L. Rodríguez-Outeiriño, D. Franco, A.E. Aranega, "PITX2 in Embryonic and Adult Myogenesis," Front Cell Dev. Biol., vol.5, pp. 46, October 2017.

[19] W. Liu, J. Selever, M.F. Lu, J.F. Martin, "Genetic dissection of Pitx2 in craniofacial development uncovers new functions in branchial arch morphogenesis, late aspects of tooth morphogenesis and cell migration," Development, vol.130, pp. 6375-6385, December 2003

[20] D.M. Martin, F.J. Probst, S.E. Fox, L.A. Schimmenti, E.V. Semina, M.A. Hefner, et al., "Exclusion of PITX2 mutations as a major cause of CHARGE association," Am. J. Med. Genet., vol. 111, pp. 27-30, July 2002.

[21] J.C. Phillips, "Four novel mutations in the PITX2 gene in patients with Axenfeld-Rieger syndrome," Ophthalmic Res., vol. 34, pp. 324-326, September 2002.

[22] N. Weisschuh, P. Dressler, F. Schuettauf, C. Wolf., B. Wissinger, E. Gramer, "Novel mutations of FOXC1 and PITX2 in patients with Axenfeld-Rieger malformations," Invest. Ophthalmol. Vis. Sci., vol. 47, pp. 3846-3852, March 2013.

[23] Johns Hopkins University. (2017, July 28) "PITX2". Available: http://www.omim.org/entry/601542?search=PITX2\%20\%20gen e\&highlight=PITX2\%20\%20gene/. c1966-2017.

[24] L.M. Moreno Uribe, S.F. Miller, "Genetics of the dentofacial variation in human malocclusion," Orthod. Craniofac. Res., vol. 18, pp. 91-99, April 2015.
[25] S. Sharma, D.S. Gupta, U.S. Pal, S.K. Jurel, "Etiological factors of temporomandibular joint disorders," Natl. J. Maxillofac. Surg., vol. 2, pp.116-119, July 2011. 